\title{
There exists high ratio of, and significant overlap between, suspected dementia and suspected depression among elderly outpatients
}

\section{Yao-Tung Lee}

Taipei Medical University-Shuang Ho Hospital

\section{Chia-Fen Tsai}

Taipei Veterans General Hospital

Yen-Kuang Lin

$$
\text { National Taiwan Sport University }
$$

Cheng-Che Shen

Taichung Veterans General Hospital

\section{Li-Kai Huang}

Taipei Medical University-Shuang Ho Hospital

\section{Mei-Ju Chi}

Taipei Medical University

Hsin-Chien Lee ( $\nabla$ ellalee@tmu.edu.tw )

Taipei Medical University

\section{Research Article}

Keywords: dementia, depression, older adults, screening, AD8, BSRS-5

Posted Date: October 12th, 2021

DOl: https://doi.org/10.21203/rs.3.rs-954632/v1

License: (9) This work is licensed under a Creative Commons Attribution 4.0 International License. Read Full License 


\section{Abstract \\ Background}

Dementia and depression have emerged as two of the major mental health concerns for elderly people, which are likely to substantially affect public health in the coming decades. Both dementia and geriatric depression are associated with increased morbidity, health care utilization, and mortality. Early detection and treatment initiation are some of the best strategies to minimize the ill effects of dementia and depression on the elderly people. To this end, large-scale screening may be the first step.

\section{Methods}

Screening of individuals $\geq 65$ years old was conducted in the outpatient department of a university hospital. Ascertainment of dementia 8 (AD8) and five-item Brief Symptom Rating Scale (BSRS-5) were used to screen for cognitive decline and depression, respectively.

\section{Findings:}

In total, 3079 elderly people completed both AD8 and BSRS-5. Patients tested positive for cognitive impairment and depression were $28.2 \%$ and $15 \%$, respectively. However, $7 \%$ were tested positive by both AD8 and BSRS- 5 and with statistical significance.

\section{Interpretation:}

The high ratio of suspected dementia and suspected depression suggested the needs and cost-effect of screening among the elderly outpatients. However, the statistically significant overlap implied potential bias when screening only one condition. Future screening program of geriatric mental health needs to consider this.

\section{Introduction}

The increase of the elderly population is a global trend. The percentage of the population aged $>65$ years in Taiwan increased remarkably from 7.1\% in 1993 to 14.6\% in 2018, making it one of the countries with a rapidly increasing aging population ${ }^{1}$. Furthermore, this percentage is expected to reach $20.7 \%$ in 2026 , which may place a serious burden on the health care system and the national health insurance costs. Dementia and depression are the most common psychiatric illnesses in elderly people. Both these conditions are chronic, impair daily living activities, and increase comorbidity, thus greatly increasing the burden to the society. 
The statistical data from World Health Organization showed that in 2015, dementia affected 47 million people worldwide, and this population is expected to increase to 75 million by 2030 and 132 million by $2050^{2}$. Alzheimer's disease (AD) is one of the most common type of dementia. A report had estimated that by 2050 in the United States, one new case of AD is expected to develop every 33 seconds, resulting in nearly 1 million new cases per year ${ }^{3}$. Because the cognitive deterioration of dementia is usually continuous which may begin with a very early stage of mild cognitive impairment $(\mathrm{MCl})$, the estimated prevalence of dementia throughout the world is variable. A recent nationwide survey in Taiwan that recruited 10,432 participants aged $\geq 65$ years revealed that the age-adjusted prevalence percentages of all-cause dementia and $\mathrm{MCl}$ were was $8.04 \%$ and $18.76 \%$, respectively ${ }^{4}$. Studies had reported that a large proportion of dementia patients have comorbid chronic diseases, contributing to increased outpatient department visits and medical burden 5,6. The huge economic load of dementia in 2010 was estimated worldwide cost to be US\$ 604 billion, equivalent to $1 \%$ of the world's gross domestic product ${ }^{7}$. A common consensus now is that early detection and treatment initiation are some of the best strategies to delay dementia progression and to preserve the patient's function level, with the subsequent benefit of less societal $\operatorname{cost}^{8}$. However, because dementia requires many years to develop, detecting the disease at an early stage inclusive of $\mathrm{MCl}$ and very mild dementia, is difficult.

Large-scale epidemiological investigations have revealed that the prevalence of depression in those aged $\geq 65$ years ranges from $1-5 \%$ for major depressive disorder and $8-16 \%$ for clinically significant depressive symptoms ${ }^{9,10}$. Besides, the prevalence of geriatric depression may be higher in some specific groups. Studies have suggested those with medical conditions will markedly have high rates of depressive symptom and that the percentages of major depression are particularly high in elderly outpatients ${ }^{10,11}$. Furthermore, it had ever been reported that the prevalence of depression among elderly people in an urban slum is as high as $80 \%$, with $45 \%$ at a risk of severe depression ${ }^{12}$. Evidences had shown that elderly people with depression are strongly associated with increased morbidity, mortality, and health care utilization ${ }^{13-15}$. Moreover, geriatric depression is also associated with increased risk of suicide and decreased physical, cognitive, and social functioning bringing the society a great burden ${ }^{10}$. Thus, early diagnosis and intervention are also important for geriatric depression.

Since early detection and treatment initiation are important for both dementia and geriatric depression, large-scale screening may be the first step for this purpose. Thus, we implemented this screening program for both dementia and depression among elderly outpatients in a university hospital.

\section{Materials And Methods}

Study Design and Participants

The study was conducted at the outpatient departments of medicine and surgery in Shuang Ho hospital, a university hospital in Northern Taiwan between October 2012 and December 2014. The participants were selected through convenience sampling; those who visited the outpatient departments and aged 
$\geq 65$ years were invited to participate in this screening program. The screening includes both cognitive impairment and depression. The inclusion criteria were: 1 . the volunteers, 2 . The patients or their informants can communicate smoothly with the interviewer, 3 . without life-threatening major illnesses. The exclusion criteria were: 1 . Deny or hesitate to participant, 2 . with difficulty in communication, 3 . cannot complete the questionnaire for both dementia and depression, 4 . with active major illness that may decrease the reliability of the answer. Ascertainment of dementia 8 (AD8) was used to screen for cognitive decline ${ }^{16}$, and five-item Brief Symptom Rating Scale (BSRS-5) was used as the screening tool for mental health, particularly depression ${ }^{17}$. A well-trained interviewer collected information regarding the age and sex of all participants, and all evaluations were conducted face-to-face with patients or their families. Reasons for declining participation were obtained. All procedures were approved by the Institutional Review Board of Taipei Medical University Hospital with IRB number to be 201211006. All methods were performed in accordance with the relevant guidelines and regulations and all participants provided informed consent before screening.

Cognitive Screening

We used AD8 to determine cognitive impairment. AD8 consists of eight items, which were answered by the informant or patient to track intraindividual functional decline attributable to cognitive impairment. AD8 has been proven to be effective in detecting even very mild dementia and is used extensively in many countries including Taiwan ${ }^{18}$. AD8 is less influenced by culture, education, sex, and age, and it requires only approximately 3 minutes for completing the screening process ${ }^{16}$. Those who scored $\geq 2$ in AD8 were considered to be suspected dementia, including very mild dementia.

\section{Depression Screening}

BSRS-5, an instrument developed by the Taiwanese ${ }^{17}$, was used to screen for depression. BSRS- 5 is a tool for screening mental health and distress severity and has been used in Taiwan since 2007. Currently, BSRS-5 is one of the most popularly instruments in Taiwan for screening depression and correlated psychological symptoms, and it had been proven to have good validity and reliability ${ }^{19}$. It is a selfadministered questionnaire and can be completed quickly in the community as well as in general medical and psychiatric settings ${ }^{20}$. BSRS- 5 consists of five items measuring the severity of five domains of psychological symptoms: (1) anxiety, (2) depression, (3) hostility (feeling easily annoyed or irritated), (4) interpersonal sensitivity (feeling inferior to other people), and (5) additional insomnia symptoms. Each symptom was rated using a 5-point Likert-type scale ranging from 0 to 4 ( $0=$ not at all; $1=$ a little bit; $2=$ moderate; 3 = severe; and 4 = extremely awful), and the total score of the five items of BSRS- 5 could be from 0 to 20. The cutoff was determined to be between 5 and 6 points. Respondents with the total score $<6$ points are considered to have good mental health ${ }^{21}$. On the contrary, people with BSRS- 5 score $\geq 6$ were considered to be suspected depression.

Statistical Analysis 
All analyses were performed using SPSS (version 12.0.1 for Windows, SPSS Inc, Chicago, IL, USA). All statistical tests were two-tailed, and $p<0.05$ indicated statistical significance. In Table 1 , we divided the continuous variable of age into 3 categories for the comparison: 65-74 years, 75-84 years and $\geqq 85$ years. In Table 2 and, we used Chi Square test to compare the effect of gender on mean score of AD8 and BSRS5. In Table 3, ANOVA was used to compare the effect of age on mean score of AD8 and BSRS-5. In Table 4 , to minimize the influence of outliers and non-normality, we chose Spearman's rank correlation coefficient analysis to examine the correlation between AD8 and BSRS- 5 scores. Independent t test was used in Table 5 and Table 6 to compare the mean score of subgroups in AD8 or BSRS- 5 screening positive participants separately.

\section{Results}

In total, 3130 elderly people participated in the program, among which 3079 received both AD8 and BSRS5. The reasons for dropping out from the study were as follows: refusing to participate, denying to answer the question regarding depression, and being in a hurry to enter the clinics because of the timing.

In Table 1, the mean age was 77.2 years with men slightly older than women. The age of the participants indicates that this is a representative group of older adults. The continuous variable of age was divided into 3 categories: young elderly (65-74 years, $N=1054,34.2 \%)$, old elderly ( $75-84$ years, $N=1390,45.1 \%$ ) and super-old elderly ( $\geqq 85$ years, $N=635,20.6 \%$ ). The number of the men is a little more than the female. According to the AD8 score, the percentage of suspected dementia is $28.2 \%$. In the meanwhile, those suspected depression on the basis of BSRS- 5 is $15.0 \%$.

As shown in figure, on the word of the screening results of AD8 and BSRS-5, the 3079 elderly people can be divided into four categories: Group 1: AD8 (-), BSRS-5 (-), $N=1973,64.1 \%$; Group 2: AD8 (+), BSRS-5 $(-), N=645,21.0 \%$; Group 3: AD8 (+), BSRS-5 (+), $N=223,7.2 \%$; and Group 4: AD8 (-), BSRS-5 (+), $N=$ $238,7.7 \%$. There exists a remarkable overlap between AD8 (+), suspected dementia, and BSRS-5 (+), suspected depression.

Table 2 presents the effect of participants' gender on the mean scores of AD8 and BSRS- 5 . The mean score for BSRS- 5 was found to be significantly higher among the female participants than among male participants. After adjustment for age, both AD8 $(p=0.01)$ and BSRS-5 $(p<0.0001)$ scores were significantly higher among the female participants than among the male participants.

The effects of age on score of AD8 and BSRS-5 are shown in Table 3. The mean score of AD8 significantly increased with age with the highest mean score in the $\geqq 85$ age group. For BSRS-5, the condition is different that the highest mean score is in the 75-84 age group and as the age getting older, the mean score of BSRS- 5 decreased.

Using Spearman's rank correlation coefficient analysis, we examined the correlation between AD8 and BSRS- 5 scores. The results are provided in Table 4 suggesting a positive correlation between the scores of AD8 and BSRS-5 and the correlation is statistic significant. 
In Table 5 and table 6, the analysis was conducted to evaluate the statistical meaning of the overlap between AD8 screening positive and BSRS- 5 screening positive. In those with the screening results of AD8 to be positive, the mean score of AD8 in BSRS-5 positive group is different from BSRS- 5 negative group and the difference has reached statistical significance. Similar result was noted in the BSRS- 5 screening positive participants that the mean score of BSRS- 5 in AD8 positive group is significantly different from AD8 negative group. These results suggested the overlap between AD 8 screening positive and BSRS- 5 screening positive to be statistically significant.

\section{Discussion}

To our knowledge, this is the first investigation involving large-scale screening for both dementia and geriatric depression simultaneously. The major findings of this study are threefold. First, there is a large proportion of suspected dementia among elderly outpatients in a university hospital. Second, the ratio of suspected depression is also notable for the same group of older adults. Third, there is a significant overlap between suspected dementia and suspected depression among this elderly group.

As shown in Table 1, there was a high AD8-positive rate (28.2\%, suspected dementia) in our study, compatible with the previous reports of using AD8 in the hospital ${ }^{22,23}$ but much higher than the AD8 screening results in the non-hospital settings ${ }^{24,25}$. There are some possible explanations for the high positive rate of AD8 in our study. First, evidences have suggested that the prevalence of dementia varies greatly based on the setting, with the estimated rates in primary care and hospital being $1.4 \%$ and $15-$ $42 \%$, respectively 26,27 , contributing to the difference in the positive rates of dementia screening. The elderly who visited the hospital must have more systemic diseases including diabetes mellitus, hypertension and dyslipidemia than the elderly in community and these age-related chronic illnesses have been suggested to increase the risk of dementia ${ }^{28}$. Besides, because our screening program was conducted in a university hospital, patients visited here may have suffered from complicated disease for a period of time that may have even higher risk of dementia. Thus, it is reasonable to predict a higher positive rate of AD8 screening in our study. Second, being hospital-based screening with AD8, the former two studies recruited participants $<60$ years old with the median age and the mean age to be 66 and 69.9 years separately. On the contrary, only those $\geq 65$ years were recruited in our study making the mean age much older than the previous investigations (77.2 years). And age is one of the established risk factors for dementia. Third, although studies have supported the effectiveness of AD8 in dementia screening, a recent Cochrane review suggested that the $A D-8$ has a higher relative sensitivity $(1.11,95 \% \mathrm{Cl} 1.02$ to $1.21)$, but lower relative specificity $(0.51,95 \% \mathrm{Cl} 0.23$ to 1.09$)$, in secondary care settings compared to community care settings ${ }^{29}$. This character may increase the positive rate of AD8 screening in the hospital settings. Fourth, besides of screening for dementia, AD8 has been reported to be effective in detecting $\mathrm{MCl}$ and very mild dementia ${ }^{18,30}$. Thus, the elderly with AD8 screening positive in our study may include some $\mathrm{MCl}$ cases. In summary, AD8 screening in hospital-based settings showed significantly higher positive rate than community-based screening. The high sensitivity of AD8 in the secondary care settings ${ }^{29}$ suggests its effect in identifying adults who may benefit from further evaluation for dementia. 
AD8 is a screening tool, the diagnostic confirmation requires more detailed assessment including laboratory tests, brain image analysis and psychological testing etc., and all of these are available in the general hospital contributing to a better diagnostic facility.

Table 1 also showed that with the screening tool of BSRS-5, $15.0 \%$ of elderly outpatients were suspected depression. Compared with previous studies of using BSRS- 5 in older adults $\geqq 65$ years, the positive rates for community and inpatients of medical or surgical conditions were $9.5 \%^{31}$ and $25.3 \%^{32}$ respectively. Our result suggested that the positive percentage for elderly outpatients was between these two rates. There used to be many studies investigating the prevalence of geriatric depression and the results vary depending on the definition of depression and on the target subsets of the elderly population. The terms used in geriatric depression include major depressive disorder, major depression, clinically significant depressive symptoms, and depressive symptom etc., which represent different severity, different definition or diverse tools of evaluation. Compared with using the DSM-V or ICD-10 criteria, the prevalence for investigations that used the depressive symptom checklist to define the clinical case of geriatric depression must be higher. And in general, the prevalence may be higher in the hospital-based settings than in the community. Along these lines, there were several rates of geriatric depression being reported before. In a community study of adults aged $\geq 65$ years, Hasin et al., have reported the prevalence of major depressive disorder to range from $1-5 \%$ at any time ${ }^{9}$. By contrast, in a review article, Blazer reported that the clinically significant depressive symptoms are present in approximately $15 \%$ of community-dwelling elderly people ${ }^{10}$. Besides, the rates of major depression among elderly people had been reported to be higher in certain subgroups, including medical outpatients (5-10\%) and medical inpatients $(10-12 \%)^{10,11}$. A study carried out in outpatient setting of geriatric clinics of tertiary care hospital using the Geriatric Depression Scale (GDS-15) showed a rate of $22 \%$ of participants scored high on the GDS $(\geq 5)$ and $18 \%$ were definitely having a depressive disorder as per ICD $10^{33}$. It seemed that among elderly outpatients, the positive rate of BSRS- 5 was lower than that of the GDS-15 but closer to the percentage of depressive disorder defined by ICD10. Further studies may be needed to get more information about the usage of BSRS- 5 in the screening of depression among elderly outpatients. In summary, the notable positive rate of BSRS- 5 among elderly outpatients in our study implies the costeffect of performing the screening of geriatric depression for this group in order to detect the disease earlier and start treatment. At the same time, before diagnosing the geriatric depression, a series of survey is needed to rule out the possible existence of other organic lesions. And the hospitals just meet the needs which reinforce the benefit of implementing the screening program in the hospital-based settings.

The analysis of the effect of the participant's gender suggested that after adjustment for age, the mean score for female is higher than male in both AD8 and BSRS- 5 and with statistical significance. The results are compatible with previous investigations indicating that female is one of the risk factors for both dementia and geriatric depression ${ }^{11,34}$. Besides, in Table 3 , the analysis about the effect of the age on AD8 and BSRS- 5 showed that the mean scores significantly increased with age in AD8 but not in BSRS- 5 . These findings are also compatible with previous reports which suggested age to be a risk factor for dementia but not for geriatric depression ${ }^{34,35}$. The analysis regarding the effect of the elderly patients' 
gender and age on AD8 and BSRS-5 are twofold. First, when elderly female with chronic illnesses is involved, clinicians should pay more attention to the tendency of both cognitive decline and depression. Second, although evidence indicates that depression becomes less common and less severe with age, the lower-severity depressive symptoms, which can also be consequential and treatable, should not be overlooked $^{36}$.

Besides of the high prevalence of suspected dementia and suspected depression among the elderly outpatients, our study furtherly suggested that there was a remarkable overlap between suspected dementia and suspected depression. In other words, to implement a screening program for both dementia and depression among elderly outpatients in a university hospital, there exists about $7 \%$ with positive results in both dementia and depression screening. Further analysis suggested that the overlap between suspected dementia and suspected depression had reached the statistical significance. These results suggested that, for the older adults in outpatient department, to perform the screening either for dementia or for geriatric depression alone may have some bias. Spearman's rank correlation analysis in our study also implied that the instruments for dementia screening and for depression screening are not completely independent. Compared with the past investigations, there used to be little study to perform the largescale screening for both dementia and geriatric depression at the same time. But this result is partially compatible with a current consensus that increasing evidence supports a linkage between dementia and depression ${ }^{37,38}$. There are some possible explanations for the significant overlap between the suspected dementia and suspected depression in our study. First, evidences have suggested a high prevalence of depressive symptoms in dementia patients. In a study of Zubenko et al., depressive symptoms were reported in $30-50 \%$ of patients with Alzheimer disease, particularly at the prodromal stage ${ }^{39}$. Furthermore, major depression was observed in $>10 \%$ of patients with Alzheimer disease and in $20 \%$ of those with vascular dementia 40,41 suggesting a high ratio of positive results in both dementia and depression screening. Second, although being an old concept, the key role of depression, especially that has not been diagnosed and without treatment, in the background of pseudo-dementia may also contribute partially to the overlap between suspected dementia and suspected depression ${ }^{42}$. Third, cognitive deterioration and depression may share some of the common etiology contributing to their cooccurrence. Several mechanisms have been proposed to explain the connection between depression and dementia of which vascular disease has been proved to be a primary link between them which is supported by the vascular depression hypothesis ${ }^{43,44}$. Evidence also indicates that ischemic damages to the brain, particularly the frontostriatal brain regions, might be a common etiologic factor for depression and cognitive dysfunction ${ }^{38,45}$. Besides, it had been proposed that increased cortisone levels, frequently observed in depression, can lead to worsening hippocampal atrophy, which is a well-known characteristic of Alzheimer disease ${ }^{38,46}$. Other possible systemic etiology includes chronic inflammatory processes 47 as well as changed levels of circulating brain-derived neurotrophic factors ${ }^{48,49}$. Consequently, our study suggested that when implementing a screening program for the elderly outpatients, both dementia and depression should be included. What should be emphasized is that AD8 and BSRS-5 are not diagnostic 
tools. Further studies are needed to clarify the real mental status of those with positive results in both screening tools.

This study has some strengths. First, past studies have focused on screening for either dementia or geriatric depression, whereas ours is the first study to perform large-scale screening for both dementia and geriatric depression simultaneously. Second, our study targeted at-risk patients in the outpatient departments of medicine and surgery whose mental health are usually poorer but are ignored. Third, AD8 and BSRS- 5 were used as screening tools, with the former capable of identifying even $\mathrm{MCl}$ and very mild cognitive impairment and the latter, being developed by the Taiwanese, have been used widely for more than ten years in Taiwan to screen depression which can minimize the culture bond. However, this study has several limitations. First, although the participants were volunteers, they were not recruited using randomized sampling from the outpatient department, which may have led to selection bias. Second, we did not separate the informant AD8 from participant AD8. Studies have suggested that the former could be more precise than the latter 50,51 . Third, the elderly patients recruited were from only one hospital, which is in a cultural and educational district of Northern Taiwan. Furthermore, patients from our university hospital may have more complex comorbid illnesses than others do and cannot represent the general condition of elderly patients in the outpatient department. Fourth, we did not collect information regarding education and comorbidities of participants which contributed to the lacking of the content in descriptive statistics. Additionally, further evaluation for differential diagnosis was lacking, which made the analysis of sensitivity and specificity unavailable.

This study of implementing a screening program for both dementia and depression in a university hospital suggest the high ratio of, and significant overlap between, suspected dementia and suspected depression among the elderly outpatients. Given the projected increase in the dementia and geriatric depression population in the coming decades, large-scale screening of elderly adults may be needed for the early detection and early intervention of these two illnesses. Overall, this study strengthens the costeffect of performing the screening in the elderly of outpatient department and the screening should include both dementia and depression. Further investigation should be conducted to elucidate the status of the cognition and emotion among the elderly outpatients especially those with both screening to be positive.

\section{Declarations}

\section{Authors' contributions}

Study conception and design: Y.T.L. and H.C.L. Acquisition of data: Y.T.L., C.F.T. and M.J.C. Analysis and interpretation of data: Y.K.L, C.C.S. and L.K.H. Draft manuscript: Y.T.L. and M.J.C. All authors had read and approved the final manuscript.

\section{Declaration of Conflicting Interests}

The authors declare that there is no conflict of interest. 


\section{Funding}

This work was supported by grants (No: 110TMU-SHH-26) from Taipei Medical University Shuang Ho Hospital.

Written informed consent for publication of the clinical details was obtained from all the patients.

\section{References}

1. National Development Council. The aging population projection in Taiwan. [Internet], 2020 9/10/2020.

2. (2017)., W. H. O. Global action plan on the public health response to dementia 2017 - 2025.. Geneva: World Health Organization, 52.

http://www.who.int/mental_health/neurology/dementia/action_plan_2017_2025/en/.

3. 2015 Alzheimer's disease facts and figures. Alzheimers Dement 11, 332-384, doi:10.1016/j.jalz.2015.02.003 (2015).

4. Sun, Y. et al. A nationwide survey of mild cognitive impairment and dementia, including very mild dementia, in Taiwan. PLoS One, 9, e100303 https://doi.org/10.1371/journal.pone.0100303 (2014).

5. Schubert, C. C. et al. Comorbidity profile of dementia patients in primary care: are they sicker? J Am Geriatr Soc, 54, 104-109 https://doi.org/10.1111/j.1532-5415.2005.00543.x (2006).

6. Poblador-Plou, B. et al. Comorbidity of dementia: a cross-sectional study of primary care older patients. BMC Psychiatry, 14, 84 https://doi.org/10.1186/1471-244x-14-84 (2014).

7. Martin Prince, D. M. P. a. D. M. G. World Alzheimer Report 2013: An analysis of long-term care for dementia 2013 Alzheimer's Disease International.

8. Leifer, B. P. Early diagnosis of Alzheimer's disease: clinical and economic benefits. J Am Geriatr Soc, 51, S281-288 https://doi.org/10.1046/j.1532-5415.5153.x (2003).

9. Hasin, D. S., Goodwin, R. D., Stinson, F. S. \& Grant, B. F. Epidemiology of major depressive disorder: results from the National Epidemiologic Survey on Alcoholism and Related Conditions. Arch Gen Psychiatry, 62, 1097-1106 https://doi.org/10.1001/archpsyc.62.10.1097 (2005).

10. Blazer, D. G. Depression in late life: review and commentary. J Gerontol A Biol Sci Med Sci, 58, $249-$ 265 https://doi.org/10.1093/gerona/58.3.m249 (2003).

11. Djernes, J. K. Prevalence and predictors of depression in populations of elderly: a review. Acta Psychiatr Scand, 113, 372-387 https://doi.org/10.1111/j.1600-0447.2006.00770.x (2006).

12. Ashe, S. \& Routray, D. Prevalence, associated risk factors of depression and mental health needs among geriatric population of an urban slum, Cuttack, Odisha. Int J Geriatr Psychiatry, 34, 17991807 https://doi.org/10.1002/gps.5195 (2019).

13. Lyness, J. M., Niculescu, A., Tu, X., Reynolds, C. F., Caine, E. D. \& 3rd \& The relationship of medical comorbidity and depression in older, primary care patients. Psychosomatics, 47, 435-439 https://doi.org/10.1176/appi.psy.47.5.435 (2006). 
14. Wei, J. et al. The association of late-life depression with all-cause and cardiovascular mortality among community-dwelling older adults: systematic review and meta-analysis. Br J Psychiatry, 215, 449-455 https://doi.org/10.1192/bjp.2019.74 (2019).

15. Han, K. M. et al. Relationship of depression, chronic disease, self-rated health, and gender with health care utilization among community-living elderly. J Affect Disord, 241, 402-410 https://doi.org/10.1016/j.jad.2018.08.044 (2018).

16. Galvin, J. E. et al. The AD8: a brief informant interview to detect dementia. Neurology, 65, 559-564 https://doi.org/10.1212/01.wnl.0000172958.95282.2a (2005).

17. Lee, M. B. et al. Development and verification of validity and reliability of a short screening instrument to identify psychiatric morbidity. J Formos Med Assoc, 102, 687-694 (2003).

18. Yang, Y. H. et al. Application of AD8 questionnaire to screen very mild dementia in Taiwanese. Am J Alzheimers Dis Other Demen, 26, 134-138 https://doi.org/10.1177/1533317510397330 (2011).

19. Lu, I. C., Jean, Y., Lei, M. C., Cheng, S. M., Wang, J. D. \& H. H. \& BSRS-5 (5-item Brief Symptom Rating Scale) scores affect every aspect of quality of life measured by WHOQOL-BREF in healthy workers. Qual Life Res, 20, 1469-1475 https://doi.org/10.1007/s11136-011-9889-4 (2011).

20. Lung, F. W. \& Lee, M. B. The five-item Brief-Symptom Rating Scale as a suicide ideation screening instrument for psychiatric inpatients and community residents. BMC Psychiatry, 8, 53 https://doi.org/10.1186/1471-244x-8-53 (2008).

21. Chen, H. C., Wu, C. H., Lee, Y. J., Liao, S. C. \& Lee, M. B. Validity of the five-item Brief Symptom Rating Scale among subjects admitted for general health screening. J Formos Med Assoc, 104, 824-829 (2005).

22. Tai, S. Y. et al. Screening dementia in the outpatient department: patients at risk for dementia. ScientificWorldJournal 2014, 138786, doi:10.1155/2014/138786 (2014).

23. Huang, L. K. et al. Dementia screening for elderly in-patients and its association with nursing care satisfaction-an observational study. Med. (Baltim), 99, e18741 https://doi.org/10.1097/md.0000000000018741 (2020).

24. Chen, C. H., Wang, L. C., Ma, T. C. \& Yang, Y. H. A walk-in screening of dementia in the general population in Taiwan. ScientificWorldJournal 2014, 243738, doi:10.1155/2014/243738 (2014).

25. Chen, G. C. et al. Vascular, Cognitive, and Psychomental Survey on Elderly Recycling Volunteers in Northern Taiwan. Front Neurol, 9, 1176 https://doi.org/10.3389/fneur.2018.01176 (2018).

26. Donegan, K. et al. Trends in diagnosis and treatment for people with dementia in the UK from 2005 to 2015: a longitudinal retrospective cohort study. Lancet Public Health, 2, e149-e156 https://doi.org/10.1016/s2468-2667(17)30031-2 (2017).

27. Jackson, T. A. et al. Challenges and opportunities in understanding dementia and delirium in the acute hospital. PLoS Med, 14, e1002247 https://doi.org/10.1371/journal.pmed.1002247 (2017).

28. Sheladia, S. \& Reddy, P. H. Age-Related Chronic Diseases and Alzheimer's Disease in Texas: A Hispanic Focused Study. J Alzheimers Dis Rep, 5, 121-133 https://doi.org/10.3233/adr-200277 (2021). 
29. Hendry, K. et al. AD-8 for detection of dementia across a variety of healthcare settings. Cochrane Database Syst Rev, 3, CD011121 https://doi.org/10.1002/14651858.CD011121.pub2 (2019).

30. Razavi, M. et al. Comparison of 2 informant questionnaire screening tools for dementia and mild cognitive impairment: AD8 and IQCODE. Alzheimer Dis Assoc Disord, 28, 156-161 https://doi.org/10.1097/wad.0000000000000008 (2014).

31. Tseng, T. J. et al. Association between health behaviors and mood disorders among the elderly: a community-based cohort study. BMC Geriatr, 19, 60 https://doi.org/10.1186/s12877-019-1079-1 (2019).

32. Liao, S. J., Wu, B. J., Liu, T. T., Chou, C. P. \& Rong, J. R. Prevalence and characteristics of suicidal ideation among 2199 elderly inpatients with surgical or medical conditions in Taiwan. BMC Psychiatry, 18, 397 https://doi.org/10.1186/s12888-018-1981-7 (2018).

33. Prakash, O., Gupta, L. N., Singh, V. B. \& Nagarajarao, G. Applicability of 15-item Geriatric Depression Scale to detect depression in elderly medical outpatients. Asian J Psychiatr, 2, 63-65 https://doi.org/10.1016/j.ajp.2009.04.005 (2009).

34. Alzheimer's \& Association 2017 Alzheimer's disease facts and figures. Alzheimer's \& Dementia, 13, 325-373 (2017).

35. Fiske, A., Wetherell, J. L. \& Gatz, M. Depression in older adults. Annu Rev Clin Psychol, 5, 363-389 https://doi.org/10.1146/annurev.clinpsy.032408.153621 (2009).

36. Judd, L. L., Schettler, P. J. \& Akiskal, H. S. The prevalence, clinical relevance, and public health significance of subthreshold depressions. Psychiatr Clin North Am, 25, 685-698 https://doi.org/10.1016/s0193-953x(02)00026-6 (2002).

37. Byers, A. L. \& Yaffe, K. Depression and risk of developing dementia. Nat Rev Neurol, 7, 323-331 https://doi.org/10.1038/nrneurol.2011.60 (2011).

38. Butters, M. A. et al. Pathways linking late-life depression to persistent cognitive impairment and dementia. Dialogues Clin Neurosci, 10, 345-357 https://doi.org/10.31887/DCNS.2008.10.3/mabutters (2008).

39. Zubenko, G. S. et al. A collaborative study of the emergence and clinical features of the major depressive syndrome of Alzheimer's disease. Am J Psychiatry, 160, 857-866 https://doi.org/10.1176/appi.ajp.160.5.857 (2003).

40. Lopez, O. L. et al. Psychiatric symptoms vary with the severity of dementia in probable Alzheimer's disease. J Neuropsychiatry Clin Neurosci, 15, 346-353 https://doi.org/10.1176/jnp.15.3.346 (2003).

41. Park, J. H. et al. Depression in vascular dementia is quantitatively and qualitatively different from depression in Alzheimer's disease. Dement Geriatr Cogn Disord, 23, 67-73 https://doi.org/10.1159/000097039 (2007).

42. Voros, V. et al. Untreated depressive symptoms significantly worsen quality of life in old age and may lead to the misdiagnosis of dementia: a cross-sectional study. Ann Gen Psychiatry, 19, 52 https://doi.org/10.1186/s12991-020-00302-6 (2020). 
43. Alexopoulos, G. S. et al. 'Vascular depression' hypothesis. Arch Gen Psychiatry, 54, 915-922 https://doi.org/10.1001/archpsyc.1997.01830220033006 (1997).

44. Alexopoulos, G. S. Vascular disease, depression, and dementia. J Am Geriatr Soc, 51, 1178-1180 https://doi.org/10.1046/j.1532-5415.2003.51373.x (2003).

45. Alexopoulos, G. S. The vascular depression hypothesis: 10 years later. Biol Psychiatry, 60, 13041305 https://doi.org/10.1016/j.biopsych.2006.09.006 (2006).

46. Wolkowitz, O. M., Epel, E. S., Reus, V. I. \& Mellon, S. H. Depression gets old fast: do stress and depression accelerate cell aging? Depress Anxiety, 27, 327-338 https://doi.org/10.1002/da.20686 (2010).

47. Sorrells, S. F. \& Sapolsky, R. M. An inflammatory review of glucocorticoid actions in the CNS. Brain Behav Immun, 21, 259-272 https://doi.org/10.1016/j.bbi.2006.11.006 (2007).

48. Angelucci, F., Brenè, S. \& Mathé, A. A. BDNF in schizophrenia, depression and corresponding animal models. Mol Psychiatry, 10, 345-352 https://doi.org/10.1038/sj.mp.4001637 (2005).

49. Murer, M. G., Yan, Q. \& Raisman-Vozari, R. Brain-derived neurotrophic factor in the control human brain, and in Alzheimer's disease and Parkinson's disease. Prog Neurobiol, 63, 71-124 https://doi.org/10.1016/s0301-0082(00)00014-9 (2001).

50. Dong, $Y$. et al. The informant $A D 8$ is superior to participant $A D 8$ in detecting cognitive impairment in a memory clinic setting. J Alzheimers Dis, 35, 159-168 https://doi.org/10.3233/jad-122026 (2013).

51. Chio, 0. I. et al. Detection of cognitive impairment using self-rated AD8 and informant-reported AD8. J Formos Med Assoc, 117, 42-47 https://doi.org/10.1016/j.jfma.2017.02.015 (2018).

\section{Tables}

Table 1: Demographic characteristics of the recruited participants 
Total

\begin{tabular}{ll}
\hline Number (N, \%) & $3079,100 \%$ \\
\hline Age (mean \pm SD) & \\
\hline Overall & $77.2 \pm 6.8$ \\
\hline Men & $78.5 \pm 6.9$ \\
\hline Women & $75.5 \pm 6.3$ \\
\hline Age, years (N, \%) & \\
\hline $65-74$ & $1054,34.2 \%$ \\
\hline $75-84$ & $1390,45.1 \%$ \\
\hline$\geqq 85$ & $635,20.6 \%$ \\
\hline Gender (N, \%) & \\
\hline Men & $1731,56.2 \%$ \\
\hline Women & $1348,43.8 \%$ \\
\hline AD8 (mean \pm SD) & $1.0 \pm 1.1$ \\
\hline$<2$ & $2211(71.8 \%)$ \\
\hline$\geqq 2$ & $868(28.2 \%)$ \\
\hline BRS-5 (mean \pm SD) & $2.4 \pm 2.7$ \\
\hline$<6$ & $2618(85.0 \%)$ \\
\hline$\geqq 6$ & $461(15.0 \%)$ \\
\hline
\end{tabular}

AD8: ascertainment of dementia 8; BSRS-5: five-item Brief Symptom Rating Scale Table 2: Effect of gender on mean score of AD8 and BSRS-5

Mean score adjusted for age (SE)

\begin{tabular}{llll}
\hline Measure & male & female & $\mathbf{P}$ \\
\hline AD8 & $1.0(0.03)$ & $1.1(0.03)$ & 0.01 \\
\hline BSRS-5 & $2.0(0.07)$ & $2.9(0.07)$ & $<0.0001$
\end{tabular}


Unadjusted mean score (SE)

\begin{tabular}{llll} 
Measure & male & female & $\mathbf{P}$ \\
\hline AD8 & $1.0(0.03)$ & $1.0(0.03)$ & 0.17 \\
\hline BSRS-5 & $2.1(0.06)$ & $2.9(0.07)$ & $<0.0001$ \\
\hline
\end{tabular}

SE: standard error; AD8: ascertainment of dementia 8; BSRS-5: five-item Brief Symptom Rating Scale Table 3: Effect of age on mean score of AD8 and BSRS-5

\begin{tabular}{lllll} 
Age group & $\mathbf{6 5 - 7 4}$ & $\mathbf{7 5 - 8 4}$ & $\geqq 85$ & P value \\
years & $\mathbf{( N = 1 0 5 4 )}$ & $\mathbf{( N = 1 3 9 0 )}$ & $(\mathbf{N}=635)$ & \\
\hline AD8 (mean (SD)) & $0.9(1.05)$ & $1.05(1.17)$ & $1.12(1.23)$ & $<0.001$ \\
\hline BSRS-5 (mean (SD)) & $2.33(2.67)$ & $2.50(2.77)$ & $2.38(2.70)$ & 0.266 \\
\hline
\end{tabular}

SD: standard deviation; AD8: ascertainment of dementia 8; BSRS-5: five-item Brief Symptom Rating Scale Table 4: The correlation between AD8 and BSRS-5

\begin{tabular}{ll}
$\begin{array}{l}\text { Spearman's rank correlation, } \\
N=3079\end{array}$ & BSRS-5 \\
\hline AD-8 & $0.26162^{\star \star}$ \\
${ }^{*} \mathrm{p}<0.001$ & \\
\hline
\end{tabular}

AD8: ascertainment of dementia 8; BSRS-5: five-item Brief Symptom Rating Scale

Table 5: Subgroup analyses of AD8 screening positive participants

\begin{tabular}{|llll|}
\hline & BSRS-5 (-) & BSRS-5(+) & \\
\hline & $(\mathrm{N}=645)$ & $(\mathrm{N}=223)$ & $P$ value \\
\hline BSRS-5 (mean (SD)) & $1.87(1.64)$ & $7.76(2.08)$ & $<0.001^{*}$ \\
\hline AD8 (mean (SD)) & $2.5(0.76)$ & $2.74(1.06)$ & $<0.001^{\star}$ \\
\hline
\end{tabular}

AD8(+), suspected dementia participants

* $P<0.05$ 
AD8: ascertainment of dementia 8; BSRS-5: five-item Brief Symptom Rating Scale

Table 6: Subgroup analyses of BSRS-5 screening positive participants

\begin{tabular}{|llll|}
\hline & AD8(-) & AD8(+) & \\
\hline & $(\mathrm{N}=238)$ & $(\mathrm{N}=223)$ & $P$ value \\
\hline BSRS-5 (mean (SD)) & $7.36(1.85)$ & $7.76(2.08)$ & $0.029 *$ \\
\hline AD8 (mean (SD)) & $0.48(0.5)$ & $2.74(1.06)$ & $<0.001^{*}$ \\
\hline
\end{tabular}

BSRS-5(+), suspected depression participants

* $P<0.05$

AD8: ascertainment of dementia 8; BSRS-5: five-item Brief Symptom Rating Scale

\section{Figures}

$\downarrow$ Drop out $1.6 \%$

$\mathrm{N}=3079$ fulfill the inclusion criteria

\begin{tabular}{llll}
\hline Group 1 & Group 2 & Group 3 & Group4 \\
AD8 (-) & AD8 (+) & AD8 (+) & AD8 (-) \\
BSRS-5 (-) & BSRS-5 (-) & BSRS-5 (+) & BSRS-5 (+) \\
N $=1973,64.1 \%$ & $\mathrm{~N}=645,21.0 \%$ & $\mathrm{~N}=223,7.2 \%$ & $\mathrm{~N} 238,7.7 \%$
\end{tabular}

Figure 1

AD8 and BSRS- 5 screening of outpatient patients $N=3130$ 